Artigo / Article

\title{
Mieloma Múltiplo e anemia
}

Multiple Myeloma and anemia

Rodolfo D. Cançado

\begin{abstract}
Anemia é uma complicação comum em pacientes com mieloma múltiplo (MM) e ocorre em mais de 2/3 dos pacientes. Anemia de doença crônica, deficiência de eritropoetina (EPO) devido à insuficiência renal e efeito mielossupressivo da quimioterapia são os principais mecanismos patofisiológicos que contribuem para o desenvolvimento de anemia no MM. Nos pacientes que obtêm remissão completa com tratamento quimioterápico, anemia usualmente se normaliza. Nos pacientes que não respondem ou apresentam recaída do mieloma, anemia freqüentemente persiste. As opções de tratamento dos pacientes anêmicos com MM incluem transfusões de hemácias e EPO recombinante humana. Essa proteína é biologicamente equivalente à EPO endógena e sua administração promove aumento dos valores de hemoglobina por tempo mais prolongado sem os riscos das transfusões de sangue. Vários estudos têm relatado melhora significante da eritropoese, redução da necessidade transfusional e melhora da qualidade de vida com o uso da EPO como tratamento a longo prazo da anemia associada ao mieloma. Nesse artigo, propomos o tratamento da anemia do MM baseado nas recomendações propostas pela Sociedade Americana de Hematologia (ASH) em conjunto com a Sociedade Americana de Oncologia Clínica (ASCO), pela Organização Européia para Pesquisa e Tratamento do Câncer (EORTC), pelo IMF (Internacional Myeloma Foundation) e pelo NCCN (National Comprehensive Cancer Network).Rev. bras. hematol. hemoter. 2007;29(1):67-76.
\end{abstract}

Palavras-chave: Anemia; mieloma múltiplo; eritropoetina.

\section{Introdução}

Anemia é definida como condição patológica decorrente da diminuição do número de glóbulos vermelhos ou da concentração da hemoglobina ocasionando diminuição da oxigenação tecidual.

Nas últimas duas décadas, grande parte do esforço dispensado concentrou-se na busca de melhor entendimento da biologia da célula tumoral bem como na procura de esquemas de tratamento que oferecessem melhores resultados e que fossem capazes de interferir no curso natural da doença neoplásica. ${ }^{1,2}$

Em contrapartida, a anemia no paciente com câncer é, de longa data, considerada como achado comum do ponto de vista de sinal e relativamente pouco importante do ponto de vista de sintoma. Ainda nos dias atuais se dá pouca importância para o tratamento da anemia nos pacientes com câncer, sobretudo nos pacientes com mieloma múltiplo (MM), uma vez que sintomas relacionados à anemia, sobretudo fadiga e letargia, são freqüentemente subestimados e subvalorizados ou atribuídos unicamente como conseqüência da doença neoplásica ou do tratamento quimioterápico instituído. ${ }^{1,3}$

Dependendo da doença neoplásica, a freqüência de anemia varia entre 20,0\% e 60,0\%. Recente estudo prospectivo analisando mais de 15.000 pacientes com câncer de 24 países europeus observou que $39 \%$ desses pacientes tinham anemia (hemoglobina < 12,0 g/dl) ao diagnóstico. Entre os pacientes que não apresentavam anemia no início do estudo, $63 \%$ tornaram-se anêmicos com o tratamento quimioterápico

Professor assistente da Disciplina de Hematologia e Oncologia da Faculdade de Ciências Médicas da Santa Casa de Misericórdia de São Paulo.

Correspondência: Rodolfo Delfini Cançado

Hemocentro da Santa Casa de São Paulo

Rua Marquês de Itú, 579 - $2^{\circ}$ andar

01223-001 - São Paulo-SP - Brasil

E-mail:rdcan@uol.com.br 
e/ou radioterápico. Apesar da elevada freqüência de anemia no paciente com câncer, apenas $40 \%$ foram tratados com eritropoetina (EPO). ${ }^{3}$

Segundo Tchekmedyian, ${ }^{4}$ estima-se que dos dez milhões de norte-americanos com câncer, 1,3 milhões desenvolvem anemia em algum momento da doença e apenas $20 \%$ desses pacientes são tratados com EPO.

\section{Sintomas e conseqüências clínicas da anemia}

Além de efeitos psicológicos que podem levar ao isolamento social e à depressão, presença de anemia pode causar inúmeros sintomas e sinais que estão relacionados no Quadro 1.

Quadro 1. Principais sintomas e sinais relacionados à anemia

Sistema Cardiovascular

Taquicardia, palpitação, dispnéia, sopro sistólico de ejeção

Aumento da área cardíaca, hipertrofia ventricular,

insuficiência cardíaca

Sistema Vascular

Diminuição da temperatura cutânea, palidez cutânea, mucosa e conjuntiva

Sistema Nervoso Central

Fadiga, confusão mental, vertigem, depressão, distúrbio da função cognitiva

Sistema Gastrointestinal

Anorexia, naúsea

Trato genital

Alterações menstruais, diminuição da libido

Sistema Imunológico

Diminuição da função dos macrófagos e das células T

Um dos sintomas mais comuns é fadiga, relatada em $60,0 \%$ a 96,0\% dos pacientes submetidos à quimioterapia, caracterizada por condição de exaustão que não melhora com repouso ou com o sono, ocasionado redução da habilidade do paciente para desenvolver suas funções e atividades diárias. $^{1}$

A intensidade desses sintomas e sinais depende sobretudo da intensidade da anemia e da condição clínica do paciente. Vale a pena ressaltar que decréscimo $\geq 2,0 \mathrm{~g} / \mathrm{dl}$ do valor da hemoglobina reduz significativamente o estado funcional do paciente (Índice de Karnovsky). ${ }^{3}$

\section{Prevalência de anemia no mieloma múltiplo}

Anemia é a complicação hematológica mais comum nos pacientes com MM ( $\pm 70 \%$ dos casos), sendo que cerca de $25 \%$ dos pacientes apresentam valor de hemoglobina menor que $8,5 \mathrm{~g} / \mathrm{dl}$. .,3,5

Professor Kyle, em 1975, analisando 869 pacientes com MM, já havia chamado a atenção demonstrando que $62 \%$ desses pacientes tinham anemia. Birgegard et al, ${ }^{3}$ analisan- do pacientes com MM e linfoma não-Hodgkin, observaram que $52 \%$ apresentavam anemia ao diagnóstico e 73\% após início do tratamento quimioterápico.

Kyle et $a l^{6}$ e Ludwig et al também relataram presença de anemia (hemoglobina $<12,0 \mathrm{~g} / \mathrm{dl}$ ) em $72 \%$ dos pacientes com MM.

\section{Patofisiologia da anemia no mieloma múltiplo}

O desenvolvimento da anemia no paciente com MM é multifatorial e inclui: infiltração da medula óssea por células neoplásicas, efeito mielossupressivo e nefrotóxico das drogas quimioterápicas; efeito nefrotóxico e sangramento gastrointestinal induzido pelo uso freqüente de antiinflamatórios não-hormonais; insuficiência renal, hemólise, deficiência de ferro, ácido fólico e vitamina $\mathrm{B}_{12}$; hemodiluição secundária à hiperparaproteinemia e tratamento radioterápico (quando utilizado em campos estendidos). ${ }^{1,3}$

Entretanto, o principal mecanismo patofisiológico responsável pela anemia no MM tem sido atribuído à condição denominada anemia de doença crônica (ADC). Trata-se de síndrome clínica que se caracteriza pelo desenvolvimento de anemia em pacientes que apresentam doença infecciosa crônica, inflamatória ou neoplásica. Essa síndrome tem como aspecto peculiar presença de anemia associada à diminuição da concentração do ferro sérico e da saturação da transferrina, e, paradoxalmente, quantidade de ferro medular normal ou aumentada. ${ }^{1,3,8}$

Dos vários mecanismos envolvidos na etiopatogenia da ADC, os três principais são: diminuição da sobrevida das hemácias, resposta medular inadequada frente à anemia e distúrbio do metabolismo do ferro. ${ }^{8}$

Diminuição da sobrevida das hemácias tem sido atribuída à hiperatividade do sistema mononuclear fagocitário desencadeado por processo infeccioso, inflamatório ou neoplásico. Tal estado hiperreativo leva à remoção precoce dos eritrócitos circulantes e, portanto, à diminuição da sobrevida das hemácias. Outros fatores como: febre (que pode lesar membrana eritrocitária), liberação de hemolisinas (em algumas neoplasias) e liberação de toxinas bacterianas podem levar à condição de hiper-hemólise. ${ }^{8}$

Resposta medular inadequada caracteriza-se pela incapacidade da medula óssea em aumentar sua atividade eritropoética suficientemente para compensar a menor sobrevida das hemácias. Isso deve-se, basicamente, à secreção inapropriadamente baixa de eritropoetina (EPO), à diminuição da resposta da medula óssea à EPO e à diminuição da eritropoese conseqüente à menor oferta de ferro à medula óssea. ${ }^{8}$

Resposta medular inadequada está diretamente relacionada à ativação dos macrófagos por linfócitos T e à liberação de citocinas inflamatórias, principalmente da interleucina-1 (IL-1) e interleucina-6 (IL-6), do fator de necrose tumoral alfa (TNF $\alpha$ ) e do interferon gama (INF $\gamma$ ), que atuam 
inibindo a proliferação dos precursores eritrocitários e, portanto, inibindo a eritropoese. A ação supressora dessas citocinas sobre a eritropoese supera a ação estimuladora da EPO resultando na diminuição da resposta da medula óssea à EPO e diminuição da eritropoese (Figura 1). ${ }^{8,9}$

Distúrbio da reutilização do ferro sob a forma de depósito deve-se ao aumento da síntese da lactoferrina promovido pela IL-1. Trata-se de proteína semelhante à transferrina, porém que compete com essa. A lactoferrina difere funcionalmente da transferrina em três importantes aspectos: tem maior afinidade pelo ferro, especialmente em $\mathrm{pH}$ mais baixos, não transfere o ferro às células eritropoéticas e é "retida" rápida e ativamente pelos macrófagos. Portanto, dificulta a mobilização do ferro de depósito e, conseqüentemente, prejudica a eritropoese. ${ }^{8,9}$

Além da lactoferrina, demonstrou-se que os linfócitos T ativados inibem a ação do INF $\gamma$, que, através da via do óxido nítrico e da transcrição do ácido ribonucléico mensageiro do receptor da transferrina, promove aumento da síntese de ferritina e dos receptores da transferrina, respectivamente, aumentando a captação e armazenamento do ferro no macrófago. Por outro lado, o linfócito T, principalmente via IL-4 e IL-13, estimula a síntese de ferritina e dos receptores da transferrina, que resulta no aumento do ferro sob a forma de depósito. ${ }^{8,9}$

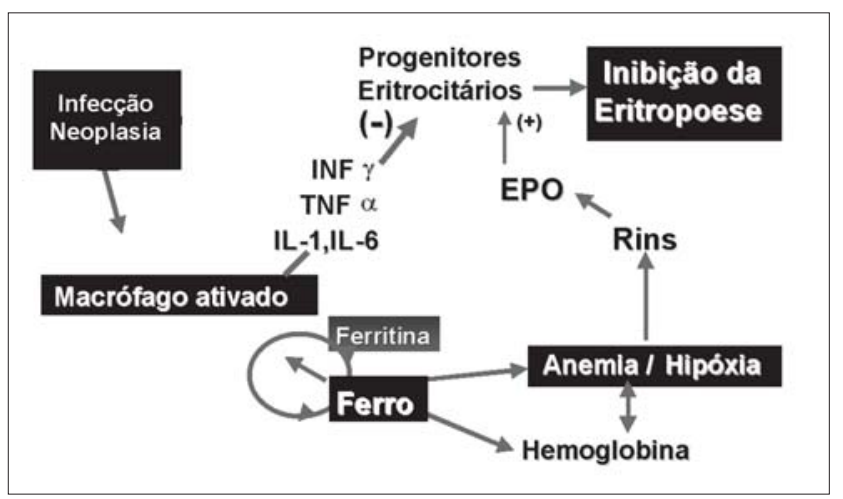

Figura 1. Representação esquemática da ação das citocinas sobre a eritropoese em pacientes com Anemia de Doença Crônica ${ }^{8,9}$ $\mathrm{EPO}=$ eritropoetina, IFN=interferon, IL=interleucina, $\mathrm{TNF}=$ fator de necrose tumoral

\section{Hepcedina e Interleucina-6 (IL-6)}

Nos últimos anos, vários estudos têm mostrado a importância da hepcedina na regulação da homeostase do ferro do organismo. Demonstrou-se, tanto em animais como em humanos, que a IL-6 age diretamente sobre os hepatócitos estimulando a síntese de hepcedina, cuja concentração plasmática pode aumentar em até 100 vezes. Essa proteína, por sua vez, atua inibindo a absorção intestinal de ferro e diminuindo a liberação de ferro presente nos macrófagos para o plasma. A Figura 2 mostra as principais etapas do

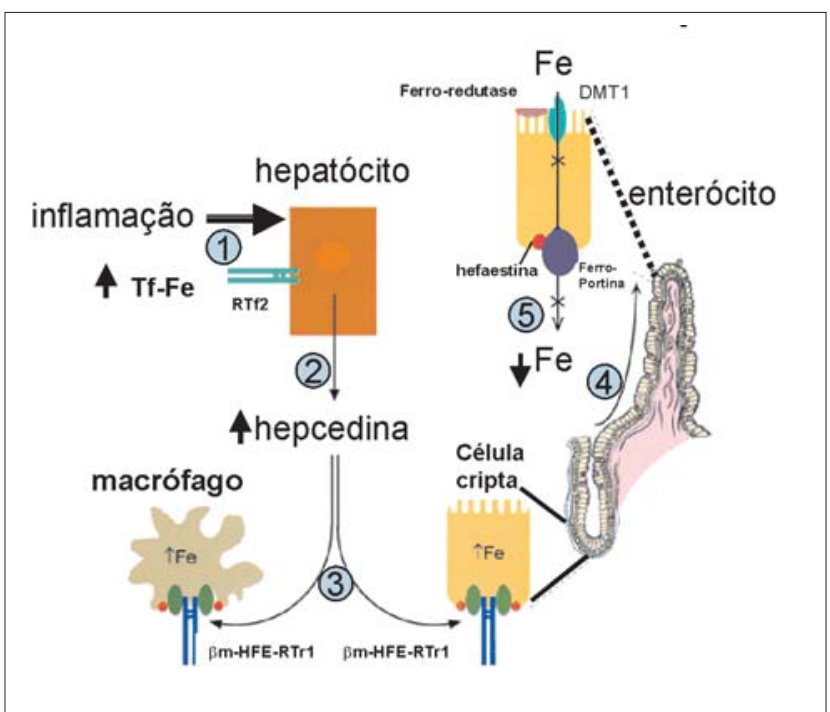

Figura 2. Representação esquemática das principais etapas do envolvimento da hepcedina na regulação do metabolismo do ferro $\mathrm{RTf}=$ =receptor da transferrina; Fe=ferro; HFE=proteína relacionada à hemocromatose; $\beta m=\beta_{2}$-microglobulina;

(1) aumento da captação do ferro ligado à transferrina através do receptor da transferrina 2 pelo fígado; (2) aumento da produção e secreção da hepcedina, que (3) interage com o complexo $\beta_{2}$-microglobulina-proteína HFE-RTf promovendo aumento da entrada de ferro nos enterócitos e aumento da retenção de ferro nos macrófagos; (4) processo de diferenciação das células da cripta intestinal. As células mais diferenciadas do vilo apresentam menor expressão de proteínas relacionadas ao transporte de ferro levando à (5) diminuição da absorção intestinal de ferro proveniente da dieta.

envolvimento da hepcedina na regulação do metabolismo do ferro ${ }^{8-10}$

Os precursores eritrocitários, sobretudo no estádio pré-basofílico ou basofílico de maturação, são altamente susceptíveis à apoptose induzida pela hiperexpressão de receptores apoptogenéticos de membrana (Fas, DR4 e DR5). Em contrapartida, eritroblastos no estádio ortocromático de maturação expressam receptores Fas ligand e tumor necrosis factor-related-apoptosis-inducing ligand (TRAIL). A ligação destes receptores de ligação com os receptores de membrana dos eritroblastos mais imaturos desencadeia a cascata das caspases e indução da apoptose desses últimos. Portanto, tanto Fas-L quanto TRAIL são fundamentais no controle intrínseco da eritropoese e esse mecanismo depende da quantidade de EPO disponível ao tecido eritropoético. ${ }^{11}$

A maturação dos eritroblastos é acompanhada do acúmulo progressivo de glicoforina A na membrana eritrocitária e depende da ação da EPO nos precursores eritrocitários e do fator-1 de transcrição eritróide (GATA-1), que estão envolvidos intrinsicamente no acúmulo de glicoforina na membrana dos eritroblastos.

Deficiência de EPO e/ou de GATA-1 diminuem ou impedem a maturação dos eritroblastos levando ao acúmulo de 
progenitores eritrocitários imaturos com elevada concentração de receptores apoptogenéticos (Fas, DR4 e DR5) e, portanto, altamente susceptíveis à apoptose.

Recentes observações demonstraram que as células plasmáticas apresentam elevada concentração de receptores Fas-L e TRAIL, e a exposição in vitro dessas células com eritroblastos imaturos induz a apoptose desses últimos através da ativação da cascata das caspases intracelular e clivagem do fator GATA-1 (Figura 3). A suplementação de EPO nessas culturas é capaz de prevenir esse processo. ${ }^{12,13}$

Entretanto, deve-se levar em consideração que é um procedimento com riscos e efeitos adversos ao paciente, tais como: transmissão de doenças infecciosas, aloimunização a antígenos celulares, reação transfusional tipo febril ou hemolítica, hemólise aguda secundária à incompatibilidade ABO, sobrecarga de ferro, imunossupressão e doença do enxerto versus hospedeiro. ${ }^{1}$

Transfusão de hemácias também pode inibir a produção endógena de EPO ocasionando diminuição da eritropoese. Esse efeito pode resultar em círculo vicioso, agravando ainda mais a dependência do paciente à transfusão alogeneica. ${ }^{1}$ Os possíveis efeitos adversos descritos anteriormente explicam porque, na maioria das vezes, a indicação desse procedimento é restrita aos pacientes com anemia mais intensa, geralmente nos casos com hemoglobina $<8,0 \mathrm{~g} / \mathrm{dl}$. $^{3}$

\section{Eritropoetina Recombinante Humana (EPO)}

O uso de EPO como fator estimulador da eritropoese é a opção terapêutica fisiológica mais importante no tratamento da anemia do paciente com doença neoplásica, mais especificamente, com MM. ${ }^{1,3,14-20}$

Trata-se de glicoproteína glicosilada de 30,4 kDa contendo 165 aminoácidos, sintetizada pelas células intersticiais renais ( $\pm 90 \%)$, além de pequena quantidade no fígado. É

\section{Tratamento}

O valor da hemoglobina aumenta e tende a se normalizar nos pacientes que obtêm remissão completa com tratamento quimioterápico. Entretanto, anemia pode persistir nos pacientes que apresentam resposta parcial ou progressão da doença, nos pacientes submetidos à múltiplos esquemas de tratamento quimioterápico e nos pacientes com disfunção renal; ou reaparecer naqueles que apresentam recaída da doença. Basicamente, o tratamento da anemia do MM consiste de transfusão de hemácias e/ou administração de eritropoetina recombinante humana. . $^{1,3,6}$

\section{Transfusão de Hemácias}

Transfusão de hemácias era a única opção efetiva no tratamento da anemia do paciente com MM e continua sendo importante recurso terapêutico nesses pacientes. Esse procedimento promove rápido aumento do valor da hemoglobina do paciente e rápida melhora dos sintomas relacionados à anemia. o principal fator modulador da eritropoese, exerce ação antiapoptótica sobre os precursores eritrocitários mais maduros e estimula a proliferação e diferenciação das células eritrocitárias na medula óssea. ${ }^{21}$

A EPO controla o dinâmico balanço entre eritropoese e catabolismo dos eritrócitos senis a fim de manter quantidade normal de glóbulos vermelhos e, em última análise, assegura o fornecimento de oxigênio a todas as células do organismo. ${ }^{21}$

Em resposta ao decréscimo da tensão de oxigênio, do aumento da afinidade do oxigênio pela hemoglobina e de qualquer estímulo capaz de reduzir a oxigenação tecidual, observa-se aumento de síntese e secreção da EPO que, no plasma, liga-se ao seu receptor específico, o qual é expresso principalmente pelas unidades formadoras de colônias eritrocítárias localizadas na medula óssea, embora, recentemente, tenha se encontrado expressão desses receptores em células de diferentes órgãos, como célula endotelial, miócito cardíaco, células neoplásicas e células neurais. ${ }^{21}$

O gene humano responsável pela codificação da EPO foi isolado em 1985. Em curto período de tempo, demons- 
trou-se a eficácia do uso dessa proteína no tratamento de anemia nos pacientes com insuficiência renal avançada e, posteriormente, no tratamento de anemia associada à doença neoplásica e à doença inflamatória ou infecciosa. ${ }^{21}$

A constatação da eficácia da EPO no tratamento da anemia associado à sua segurança e tolerabilidade contribuíram para consolidar o uso dessa droga como importante recurso terapêutico nos pacientes com anemia.,3,14-20

\section{Estudos clínicos com EPO no tratamento da anemia no mieloma múltiplo}

Os principais estudos clínicos prospectivos avaliando eficácia e segurança da EPO no tratamento da anemia em pacientes com MM estão apresentados na Tabela 1.

Tabela 1. Principais estudos clínicos com a utilização de EPO em pacientes com Mieloma Múltiplo.

\begin{tabular}{|c|c|c|c|c|c|c|}
\hline \multirow{2}{*}{ Autor $^{T I P O}$ DE ESTUDO } & \multirow{2}{*}{$\begin{array}{l}\text { Tipo de } \\
\text { EPO }\end{array}$} & \multirow{2}{*}{$\mathrm{N}$} & \multirow{2}{*}{$\begin{array}{l}\text { Dose } \\
\text { Ul/Kg }\end{array}$} & \multirow{2}{*}{$\begin{array}{l}\text { Critério de } \\
\text { Resposta }\end{array}$} & \multicolumn{2}{|c|}{$\begin{array}{c}\text { Taxa de } \\
\text { Resposta (\%) }\end{array}$} \\
\hline & & & & & EPO & Placebo \\
\hline $\begin{array}{l}\text { Ludwig }^{22} \\
\text { Fase II, NR }\end{array}$ & Alfa & 13 & $150-300$ & $\geq 2,0 \mathrm{~g} / \mathrm{dl}$ & 85 & - \\
\hline $\begin{array}{l}\text { Barlogie }^{23} \\
\text { Fase II, NR }\end{array}$ & Alfa & 28 & 150 & $\geq 2,0 \mathrm{~g} / \mathrm{dl}$ & 78 & - \\
\hline $\begin{array}{l}\text { Garton }^{16} \\
\text { Fase II, NR }\end{array}$ & Alfa & 21 & 300 & Hto $>38 \%$ & 60 & 0 \\
\hline $\begin{array}{l}\text { Cazzola }^{17} \\
\text { R, controlado, } \\
\text { multicêntrico }\end{array}$ & Beta & 84 & 1 a $10 \mathrm{mil} \mathrm{UI}$ & $\geq 2,0 \mathrm{~g} / \mathrm{dl}$ & 61 & 7 \\
\hline $\begin{array}{l}\text { Osterborg }^{19} \\
\text { Fase III, R }\end{array}$ & Beta & 65 & 2 a $10 \mathrm{mil} \mathrm{UI}$ & $\geq 2,0 \mathrm{~g} / \mathrm{dl}$ & 60 & 20 \\
\hline $\begin{array}{l}\text { Littlewood }^{2} \\
\text { Fase III, R } \\
\text { Fase III, duplo-cego, } \\
\text { placebo controlado }\end{array}$ & Alfa & 58 & $150->300$ & $\Pi \mathrm{Tx}$ & 55 & 11 \\
\hline $\begin{array}{l}\text { Dammacco15 } \\
\text { Fase III, duplo-cego, } \\
\text { placebo controlado }\end{array}$ & Alfa & 145 & $150->300$ & $\geq 2,0 \mathrm{~g} / \mathrm{dl}$ & 58 & 23 \\
\hline $\begin{array}{l}\text { Osterborg }{ }^{24} \\
\text { Fase III, R, duplo-cego, } \\
\text { placebo-controlado }\end{array}$ & Beta & 106 & $150->300$ & $\geq 2,0 \mathrm{~g} / \mathrm{dl}, \mathrm{ITx}$ & 67 & 27 \\
\hline $\begin{array}{l}\text { Dammacco }^{25} \\
\text { Fase III, } \mathrm{R}\end{array}$ & Alfa & 71 & $150-300$ & $\geq 2,0 \mathrm{~g} / \mathrm{dl}$ & 75 & - \\
\hline
\end{tabular}

NR=não randomizado; $R=$ randomizado; ITx=independência de transfusão de hemácias. Embora alguns estudos clínicos tenham incluído outras doenças linfoproliferativas, o número de pacientes da tabela refere-se apenas aos doentes com MM.

A taxa de resposta global dos pacientes com MM tratados com EPO que apresentam aumento de pelo menos 2,0 g de hemoglobina é da ordem de 60\%. Além disso, esses pacientes, na maioria das vezes, apresentam redução da necessidade transfusional e melhora da qualidade de vida. ${ }^{26}$

\section{Benefícios e limitações da EPO}

Bohlius et $a^{27}$ conduziram a mais ampla metanálise avaliando o impacto da EPO em pacientes com doença maligna, que incluiu 27 estudos clínicos controlados e randomizados envolvendo 3.287 pacientes.

Essa metanálise mostrou que o tratamento com EPO promove aumento significativo da concentração da hemoglobina (> 2,0 g/dl após, pelo menos, quatro semanas de tratamento) em número expressivo de pacientes, diminuição da necessidade transfusional (mais proeminente nos pacientes com tumor sólido do que em pacientes com neoplasia hematológica ou síndrome mielodisplásica) e melhora da qualidade de vida determinada pela melhora dos sintomas relacionados à anemia, como fraqueza, depressão, ansiedade, produtividade e do estado funcional do paciente. ${ }^{27}$

Gabrilove et $a l^{28}$, avaliando a resposta de pacientes tratados com EPO (epoetina alfa 40.000 IU/semana), observaram aumento da hemoglobina $(\geq 2,0 \mathrm{~g} / \mathrm{dl})$ ou obtenção de hemoglobina de $12,0 \mathrm{~g} / \mathrm{dl}$ sem transfusão em $68 \%$ dos casos.

Entretanto, esses resultados são obtidos em cerca de 55\% a 72\% dos pacientes, denominados respondedores à EPO. Porcentagem considerável de pacientes não respondem à EPO por razões ainda não muito bem conhecidas. Esse aspecto é muito importante, no sentido de se identificarem fatores preditores de resposta à EPO otimizando a seleção dos pacientes com maiores chances de resposta a esse medicamento. ${ }^{27}$

Em relação à sobrevida global dos pacientes tratados com EPO, 19 estudos clínicos randomizados incluindo 2.865 pacientes com câncer observaram aumento expressivo da sobrevida global dos pacientes, embora do ponto de vista estatístico a diferença observada não tenha sido significante. ${ }^{27}$

Quanto à qualidade de vida dos pacientes tratados com EPO, metanálise avaliando 23 estudos clínicos randomizados incluindo 11.459 pacientes com câncer observaram melhora significativa da qualidade de vida, enfatizando a importância do tratamento da anemia desses pacientes. ${ }^{29}$

\section{Efeitos colaterais e possíveis efeitos na biologia da célula tumoral}

O tratamento com EPO dos pacientes com MM é geralmente bem tolerado. Aproximadamente 15\% de todos os pacientes referem dor ou eritema leve no local da aplicação. Efeitos adversos graves são raros e não têm sido relatados em pacientes com MM.

Bohllius et al, ${ }^{27}$ estudando possíveis efeitos colaterais da EPO, incluindo eventos trombóticos, hipertensão arterial, 
hemorragia ou trombocitopenia, rash cutâneo, irritação, prurido, crise convulsiva, não constataram aumento significante do risco relativo de qualquer uma dessas complicações nos pacientes tratados com EPO.

Recente metanálise avaliando 32 estudos randomizados, incluindo 2.662 pacientes, demonstrou risco aumentado em 1,32 vezes de complicações tromboembólicas em pacientes anêmicos com câncer submetidos a tratamento com EPO. Em nenhum desses estudos analisados houve menção sobre possível influência da EPO no curso clínico do MM, indicando que o tratamento com EPO também é seguro em termos de qualquer influência negativa quanto à progressão da doença ou quanto ao prognóstico do paciente com MM tratado com EPO. ${ }^{30}$

Pacientes tratados com EPO têm maior risco de desenvolver hipertensão arterial. Portanto, a pressão arterial deve ser monitorada em todos os pacientes, sobretudo naqueles com insuficiência renal, particularmente durante o início da terapia com EPO.

Aplasia seletiva da série vermelha (PRCA, pure red cell aplasia) é um tipo raro de anemia caracterizada por decréscimo rápido e progressivo da hemoglobina (aproximadamente $1,0 \mathrm{~g} / \mathrm{dl}$ por semana), diminuição significativa dos precursores eritropoéticos na medula óssea e reticulocitopenia $(<10.000 / \mu \mathrm{ll}){ }^{21}$

PRCA pode ser congênita ou induzida por vários fatores como infecção, doença neoplásica, timoma, doença linfoproliferativa, doença auto-imune e por droga (incluindo insulina e interferon). ${ }^{21}$

Até 1998 foram poucos os casos relatados de PRCA. Entre 1998 e 2003 foram relatados cerca de duzentos casos de PRCA em pacientes com insuficiência renal crônica (IRC) tratados com o mesmo tipo de EPO (nenhum caso em paciente com câncer).

Esses pacientes receberam tratamento imunossupressor e 2/3 deles apresentaram recuperação da eritropoese. Acredita-se que isso seja devido à formação de anticorpos contra EPO, que também apresentam reação cruzada com a EPO endógena, levando à inibição da eritropoese, associado à desnaturação parcial da EPO provavelmente provocada pela resposta imune. ${ }^{21}$

Acredita-se que a baixa incidência de PRCA nos pacientes com câncer deve-se à alteração do sistema imunológico ou esteja relacionada à imunossupressão induzida por drogas citotóxicas. Além do fato de que o uso de EPO em pacientes com câncer é muito inferior ao uso dessa droga nos pacientes com IRC.

Mitelman et a ${ }^{31}$ observaram melhora significativa de alguns pacientes com doença refratária em termos de redução da massa tumoral indicando possível efeito antimieloma da EPO. Esse efeito foi comprovado posteriormente através de estudos experimentais em animais, demonstrando redução da massa tumoral da ordem de $30 \%$ a $60 \%$ e maior sobrevida.

\section{Fatores preditores de resposta à EPO}

Levando-se em consideração o elevado custo do tratamento com EPO e que $50 \%$ a $60 \%$ dos pacientes apresentam resposta satisfatória com essa droga, vários estudos procuraram identificar parâmetros ou fatores preditores de resposta favorável ao tratamento com EPO a fim de otimizar a seleção dos pacientes, ou seja, identificar os possíveis melhores candidatos a esse tipo de tratamento. Basicamente, os dois principais critérios de resposta são produção endógena de EPO e determinação da resposta hematopoética. ${ }^{1,3}$

Dosagem baixa de EPO $(<100 \mathrm{mU} / \mathrm{ml})$ ao diagnóstico ou baixa relação entre EPO esperada e EPO observada $(<1,0)$, aumento da hemoglobina de 0,3 a $0,5 \mathrm{~g} / \mathrm{dl}$, aumento dos receptores solúveis da transferrina (>25\%) ou aumento do número de reticulócitos ( $>40.000 / \mu l$ ), após duas semanas de tratamento com EPO, são parâmetros preditores de resposta positiva à EPO em mais de $90,0 \%$ dos casos. Em contrapartida, nos pacientes que apresentam aumento menor que $0,5 \mathrm{~g} / \mathrm{dl}$ após duas semanas de tratamento, ou que apresentam valor de EPO ao diagnóstico $>100 \mathrm{mU} / \mathrm{ml}$, a chance de resposta insatisfatória ao tratamento com EPO é de $93 \% .^{7,17}$

A combinação de relação EPO baixa e aumento dos receptores de transferrina provê probabilidade de resposta favorável da ordem de $88 \%$. O aumento da contagem de reticulócitos, só é um bom fator preditor nos pacientes que não estejam recebendo tratamento quimioterápico. Nos pacientes em tratamento quimioterápico, esse fator passa a ter mais valor se analisado após quatro semanas, e não duas, de tratamento com EPO. O aumento dos reticulócitos pode ter significado ainda maior se combinado com aumento de hemoglobina de pelo menos $1,0 \mathrm{~g} / \mathrm{dl}$ após quatro semanas de tratamento com EPO.

Osterborg et al, ${ }^{18}$ estudando pacientes com MM, linfoma não-Hodkgin e leucemia linfóide crônica, e incluindo apenas pacientes com EPO endógena menor que $100 \mathrm{mU} / \mathrm{ml}$, demonstraram que contagem de plaquetas $\geq 100.000 / \mu \mathrm{l} \mathrm{e}$ baixo requerimento de transfusão de hemácias previamente ao estudo ( $\leq 2$ unidades/mês) são os fatores preditores mais importantes associados a baixo risco de falha de tratamento.

Embora esses fatores preditores possam auxiliar na decisão do tratamento com EPO, ainda é difícil prever com maior segurança quais os melhores respondedores à EPO.

\section{Diretrizes para o tratamento da anemia no mieloma múltiplo}

As diretrizes para o tratamento da anemia nos pacientes com MM basearam-se nas principais recomendações de quatro importantes organizações médicas ligadas à hematologia e à oncologia clínica, ou seja, no consenso propos- 
to conjuntamente pela American Society of Hematology (ASH) e American Society of Clinical Oncology (ASCO) ${ }^{32}$ em 2002, no consenso proposto pela European Organization for Research and Treatment of Cancer (EORTC) ${ }^{33} \mathrm{em}$ 2003, e nas recomendações propostas pelo IMF (Internacional Myeloma Foundation) ${ }^{5}$ em 2003 e pelo NCCN (National Comprehensive Cancer Network $)^{34} \mathrm{em} 2006$.

Os três tipos de EPO recombinante humana disponíveis para uso clínico são: epoetina alfa, epoetina beta e darbopoetina..$^{35-40}$ Nesse capítulo, a denominação EPO refere-se à epoetina alfa ou à epoetina beta.

\section{Quando considerar tratamento com EPO?}

Tratamento com EPO está indicado em todo paciente com hemoglobina $\leq 10,0 \mathrm{~g} / \mathrm{dl}$ e, particularmente, nos pacientes com insuficiência renal. Transfusão de hemácias deve ser considerada dependendo da intensidade da anemia e das condições clínicas do paciente (comorbidades).

Pacientes com valor de hemoglobina entre 10,0 g/dl e 12,0 g/dl também são candidatos ao tratamento com EPO dependendo das condições clínicas do paciente (comorbidades), da intensidade dos sintomas relacionados à anemia e/ou em pacientes que apresentam diminuição progressiva do valor da hemoglobina (exemplo, redução de 1,0 a 2,0 g/dl do valor da hemoglobina entre o término do primeiro ciclo de quimioterapia e o início do segundo ciclo).

A administração concomitante de EPO é efetiva no paciente em tratamento quimioterápico uma vez que a quimioterapia induz à apoptose tanto das células neoplásicas quanto dos precursores eritrocitários normais, e a EPO diminui ou bloqueia a apoptose desses últimos.

Para pacientes dependentes de transfusão de hemácias, EPO pode ser iniciada concomitantemente à transfusão.

Não se recomenda uso profilático de EPO para prevenir anemia em pacientes em tratamento quimioterápico e/ou radioterápico com valores normais de hemoglobina no início do tratamento.

Pacientes idosos apresentam os mesmos benefícios do tratamento com EPO que os pacientes mais jovens.

Em pacientes submetidos ao transplante autólogo de células-tronco hematopoéticas, o uso de EPO não tem eficácia comprovada e não deve ser recomendado de rotina.

Em pacientes submetidos ao transplante alogênico de células-tronco hematopoéticas, o uso de EPO tem resultados limitados e pode ser recomendada caso a caso.

\section{Principais objetivos do tratamento com EPO}

Os dois principais objetivos do tratamento com EPO são melhora da qualidade de vida e prevenção de transfusão de hemácias. Não está indicado uso de EPO com o objetivo de melhorar a sobrevida do paciente ou melhorar a resposta ao tratamento uma vez que não há evidência clínica que suporte essas indicações.

\section{Fatores preditores de resposta favorável à EPO}

Dosagem sérica de EPO endógena menor que $100 \mathrm{mU} / \mathrm{ml}$, contagem de plaquetas $\geq 100.000 / \mu l$ e baixo requerimento de transfusão de hemácias previamente ao estudo ( $\leq 2$ unidades/mês) são os fatores preditores mais importantes associados à baixo risco de falha de tratamento.

\section{Que EPO e que dose utilizar?}

Epoetina alfa e epoetina beta apresentam eficácia e segurança semelhantes.

EPO deve ser iniciada na dose de $150 \mathrm{UI} / \mathrm{Kg}$ três vezes por semana, que corresponde a $10.000 \mathrm{U}$ três vezes por semana ou dose de 30.000 (EPO alfa) e 40.000U (Epoetina beta) uma vez por semana por, pelo menos, quatro semanas, ou até obtenção de hemoglobina $\geq 12,0 \mathrm{~g} / \mathrm{dl}$.

A administração semanal de darbopoetina alfa $(2,25 \mu \mathrm{g} / \mathrm{kg})$ pode ser recomendada. Biologicamente, $1 \mu \mathrm{g}$ de darbopoetina corresponde a 200 U de EPO. Até o momento, há evidência limitada que suporte o uso de darbopoetina alfa a cada 2, 3, ou 4 semanas.

\section{Quando aumentar a dose da EPO?}

Para os pacientes sem resposta satisfatória, ou seja, incremento menor que 1,0 ou 2,0 g/dl após seis a oito semanas de tratamento, lembrando da importância da investigação de deficiência de ferro, deve ser considerado aumento da dose de EPO para $300 \mathrm{UI} / \mathrm{kg}$, ou seja, 20.000U três vezes por semana ou $60.000 \mathrm{U}$ uma vez por semana. Após quatro a seis semanas com essa dose, a administração de EPO deve ser descontinuada nos pacientes que não obtiveram incremento do valor da hemoglobina.

Término e manutenção do tratamento com EPO

A partir da obtenção de hemoglobina $\geq 12,0 \mathrm{~g} / \mathrm{dl}$, a administração de EPO deve ser ajustada (redução de $25 \%$ a $50 \%$ com o objetivo de manter a hemoglobina $\pm 12,0 \mathrm{~g} / \mathrm{dl}$ ) ou descontinuada nos pacientes com hemoglobina maior que $14,0 \mathrm{~g} / \mathrm{dl}$. Nesses casos, o tratamento pode ser reiniciado com dose menor, se o valor de hemoglobina diminuir e atingir valores abaixo de 10,0 g/dl.

\section{Avaliação da eficácia da resposta à EPO}

Com relação à eficácia da resposta ao tratamento com EPO, resposta satisfatória é definida como aumento $\geq 2,0 \mathrm{~g} / \mathrm{dl}$ do valor inicial da hemoglobina (observada em pelo menos 50\% dos pacientes), diminuição do número de transfusões ou, preferencialmente, quando o paciente se torna independente de transfusão de hemácias ( \pm 80\% dos pacientes).

\section{Fatores que interferem na resposta à EPO e devem ser investigados}

Antes de iniciar o tratamento com EPO, deve-se ava- 
liar o paciente com o intuito de se afastar outras causas de anemia não associadas com a falta de eritropoetina.

Pacientes com ferritina sérica menor que $100 \mathrm{ng} / \mathrm{ml} \mathrm{e}$ saturação de transferrina $<20 \%$ antes do início do tratamento freqüentemente apresentam resposta pior que a esperada. Nesses casos, recomenda-se a reposição de ferro oral (100 a $200 \mathrm{mg}$ de ferro/dia) ou, preferencialmente, devido à ação inibidora da hepcedina na absorção intestinal de ferro, via intravenosa com sacarato de hidróxido de ferro III (100 a $200 \mathrm{mg}$ de ferro uma a duas vezes por semana).

Outras situações que podem prejudicar a resposta à EPO são: deficiência de folato, hipotireoidismo e doença infecciosa ou inflamatória, que, portanto, devem ser investigadas e tratadas quando presentes.

\section{Efeitos adversos da EPO}

Observa-se aumento do risco de evento tromboembólico em pacientes tratados com EPO. Esse efeito parece estar relacionado com o valor de hemoglobina atingido e com a rapidez do aumento da hemoglobina.

A prevalência de trombose é menor que 5\% nos pacientes com MM em uso de talidomida, de $10 \%$ a $15 \%$ quando esses medicamentos são utilizados em associação com dexametasona e de cerca de $30 \%$ nos pacientes tratados com talidomida e quimioterapia. Portanto, a indicação de EPO nesses pacientes deve ser muito bem avaliada e essa complicação monitorada adequadamente.

Pacientes tratados com EPO têm maior risco de hipertensão arterial e de aplasia seletiva de série vermelha.

\section{Considerações finais}

A introdução de esquemas terapêuticos mais eficazes no tratamento dos pacientes com MM, mudanças socioculturais ocorridas na sociedade moderna bem como mudanças em relação à própria percepção do sofrimento humano e à importância da qualidade de vida, associados à introdução da eritropoetina recombinante como tratamento da anemia, são fatores que têm influenciado positivamente e contribuído para o reconhecimento da anemia como indicador de pior prognóstico em relação à doença e indicador de pior qualidade de vida em relação ao paciente.

O objetivo principal da elaboração destas diretrizes é nortear e não cercear os médicos envolvidos no tratamento da anemia do MM. Diante da complexidade e dificuldades que envolvem os procedimentos médicos no Brasil, sobretudo no âmbito de atendimento através do sistema único de saúde, e tendo em vista o elevado custo do tratamento com EPO, há de se levar em consideração o bom senso e o senso crítico dos profissionais médicos no sentido de analisar cada paciente individualmente, de modo que condutas padronizadas nem sempre respondem ou nem sempre estão disponíveis às necessidades de cada situação clínica.

\section{Recomendações}

- $\quad$ Anemia é a complicação hematológica mais comum nos pacientes com MM ( $\pm 70 \%$ dos casos).

- $\quad$ Aproximadamente $50 \%$ e $25 \%$ dos pacientes com MM apresentam, ao diagnóstico, concentração de hemoglobina menor que $10,5 \mathrm{~g} / \mathrm{dl}$ e $8,5 \mathrm{~g} / \mathrm{dl}$, respectivamente.

- $\quad$ O valor de hemoglobina pode se normalizar nos pacientes que obtêm remissão completa com tratamento adequado (corticosteróides, etc.)

- Anemia geralmente persiste nos pacientes com:

a. resposta parcial ou progressão da doença

b. múltiplos esquemas de tratamento quimioterápico prévios

\section{c. insuficiência renal}

- Tratamento com eritripoetina tem como objetivos básicos:

a. aumento da hemoglobina (aumento ${ }^{3} 1 \mathrm{~g} / \mathrm{dl}$ em $60 \%$ dos casos) e redução dos sintomas e sinais relacionados à anemia.

b. redução da necessidade de transfusão de hemácias.

c. melhorar a qualidade de vida do paciente.

d. Transfusão de hemácias deve ser considerada dependendo da intensidade da anemia e das condições clínicas do paciente (comorbidades)

- Quando considerar eritropoetina?

a. hemoglobina igual ou inferior à $10 \mathrm{~g} / \mathrm{dl}$, sobretudo em pacientes sintomáticos, com comorbidades e com insuficiência renal.

b. importante excluir deficiência de ferro e outras causas de anemia: deficiência de folato, deficiência de vitamina B12 e hipotireoidismo, infecção, etc..

- Que dose de eritropoetina (alfa ou beta) utilizar e por quanto tempo?

a. $10.000 U \mathrm{I}( \pm 150$ a $200 \mathrm{UI} / \mathrm{Kg}$ ) subcutânea três vezes por semana ou 30.000 UI a 40.000 UI subcutânea uma vez por semana

b. pelo menos, quatro semanas, ou até obtenção de hemoglobina igual ou maior que $12 \mathrm{~g} / \mathrm{dl}$ (recomenda-se valor máximo de $13 \mathrm{~g} / \mathrm{dl}$ )

- Considerar os efeitos colaterais da eritropoetina:

a. hipertensão arterial

b. maior risco de evento tromboembólico, principalmente quando associada à talidomida e dexametasona/quimioterapia. 


\begin{abstract}
Anemia is a common complication in patients with multiple myeloma (MM) occurring in more than two thirds of all patients. Anemia of chronic diseases, erythropoietin (EPO) deficiency due to renal impairment and the myelosuppressive effect of chemotherapy are the most important pathophysiological mechanisms contributing to the development of anemia in MM. In patients who achieve complete remission after chemotherapy, anemia usually normalizes. Non-responders and relapsing myeloma patients often continue to suffer from anemia. Treatment options for anemic myeloma patients include red blood cell transfusions and recombinant human EPO. This protein is biologically equivalent to the human endogenous hormone EPO, and its application leads to an increase in hemoglobin levels over an extended time without the risks presented by blood transfusions. Several studies reported a significant improvement of erythropoiesis, reduction in transfusion need, and improved quality of life by using EPO as long-term treatment of myeloma-associated anemia. In this article we propose the treatment of myeloma-associated anemia based on recommendations by the American Society of Hematology (ASH) and American Society of Clinical Oncology (ASCO); European Organisation for Research and Treatment of Cancer (EORTC); International Myeloma Foundation (IMF) and the National Comprehensive Cancer Network (NCCN). Rev. bras. hematol. hemoter. 2007;29(1):67-76.
\end{abstract}

Key words: Anemia; multiple myeloma; erythropoetin.

\section{Referências Bibliográficas}

1. Ludwig H, Pohl G, Osterborg A. Anemia in Multiple Myeloma. Clin Adv Hematol Oncol 2004;2:233-241.

2. Littlewood TJ, Bajetta E, Nortier JW, et al. Epoetin Alfa Study Group. Effects of Epoietin Alfa on hematologic parameters and quality of life in câncer patients receiving nonplatinum chemotherapy: results of a randomized, double-blind, placebocontrolled trial. J Clin Oncol 2001;19:2.865-74.

3. Birgegard G, Aapro MS, Bokemeyer C, et al. Cancer-related anemia: pathogenesis, prevalence and treatment. Oncology 2005;68:3-11.

4. Tchekmedyian NS: Anemia in cancer patients: significance, epidemiology and current therapy. Oncology (Hungtigt) 2002; $16: 17-24$

5. Durie BGM, Kyle RA, Belch A, et al. Myeloma management guidelines: a consensus report from the Scientific Advisors of the International Myeloma Foundation. The Hematology Journal, 2003;4:379-398.

6. Kyle RA, Gertz MA, Witzig TE, et al. Review of 1027 patients with newly diagnosed multiple myeloma. Mayo Clinic Proc 2003; 78:21-33.

7. Ludwig H, Belle VS, Lee-Barrett P, et al. The European Cancer Anaemia Survey (ECAS): a large, multinational, prospective survey defining the prevalence, incidence, and treatment of anaemia in cancer patients. Eur J Cancer 2004;40:2.293-2.306.

8. Weiss G \& Goodnough LT. Anemia of chronic disease. N Engl J Med, 2005;352:1.011-23.

9.Andrews NC. Anemia of inflammation: the cytokine-hepcidin link. J Clin Invest 2004;113:1.251-1.253.
10. Ganz T. Hepcidin, a key regulator of iron metabolism and mediator of anemia of inflammation. Blood 2003;102:783-788.

11. Zamai L, Secchiero P, Pierpaoli S, et al. TNF-related apoptosisinducing ligand (TRAIL) as a negative regulator of normal human erythropoiesis. Blood 2000;95:3.716-3.724.

12. De Maria R, Testa U, Luchetti L, et al. Apoptotic role of Fas/Fas ligand system in the regulation of erythropoiesis. Blood 1999; 93:796-803.

13. Silvestris F, Tucci M, Cafforio P, et al. Fas-L up-regulation by highly malignant myeloma plasma cells: role in the pathogenesis of anemia and disease progression. Blood 2001;97:1.155-1.164.

14. Cazzolla M, Mercuriali F, Brugnara C. Use of recombinant human erythropoetin ouside the setting or uremia. Blood 1997;89: 4.248-67.

15. Dammacco F, Castoldi G. Rodjer S. Efficacy of epoetin alfa in the treatment of anemia of multiple myeloma. Br J Haematol 2001; 113:172-9.

16. Garton JP, Gertz MA, Witzig Th E, et al. Epoietin alfa for the treatment of the anemia of multiple myeloma. A prospective, randomized, placebo-controlled double blind trial. Arch Int Med 1995;155:2.069-74.

17. Cazzolla M, Messinger D, Battistel V, et al. Recombinant human erythropoietin in the anemia assoaciated with multiple myeloma and non-Hodgkins lymphoma: Dose finding and identification of predictors of response. Blood 1995;86:4.446-53.

18. Osterborg A, Boogaerts MA, Cimino R, et al. Recombinant human erythropoetin in transfusion-dependent patients with multiple myeloma and non-Hodgkins lymphoma. A randomized multicenter study. Blood 1996;87:2.675-82.

19. Österborg A, Boogaerts MA, Cimino R, et al. For the European Study Group of Erythropoietin (Epoietin Beta). Treatment in multiple myeloma and non-Hodgkin's lymphoma. Recombinant human erythropoietin in transfusion-dependent patients with multiple myeloma and non-Hodgkin's lymphoma. A randomized multicenter study. Blood 1996;87:2.675-82.

20. Engert A. Recombinant human erythropoietin in oncology: current status and further developments. Ann Oncol 2005; $16: 1.584-1.595$.

21. Kaushansky K. Lineage-specific hematopoietic growth factors. N Engl J Med 2006;354:2.034-43.

22. Ludwig H, Blade J, Dammacco F, et al. Management of diseaserelated anemia in patients with multiple myeloma or chronic lymphocytic leukemia: epoetin treatment recommendations. Hematol J 2002;3:121-130.

23. Barlogie B. Treatment of the anemia of multiple myeloma: the role of recombinant human erythropoietin. Semin Hematol 1993; 30:25-27.

24. Österborg A, Brandberg Y, Molostova V, et al. Randomized, doubleblind, placebo-controlled trial of G, Kyle R.A. Epoietin alfa for the treatment of the anemia of multiple recombinant human erythropoietin (Epoietin Beta) in hematological myeloma. A prospective, randomized, placebo-controlled double malignancies. J Clin Oncol 2002;20:2.486-94

25. Dammacco F, Silvestris F, Castoldi GL, et al. The effectiveness and tolerability of epoetin alfa in patients with multiple myeloma refractory to chemotherapy. Int J Clin Lab Res 1998;28:127-134.

26. Seidenfeld J, Piper M, Flamm C, et al. Epoetin treatment of anemia associated with cancer therapy: A systematic review and meta-analysis of controlled clinical trials. J Natl Cancer Inst 2001; 93:1.204-1.214.

27. Bohlitus J, Langensiepen S, Scharzer G, et al. Recombinant human erythropoietin and overall survival in cancer patients: results of a comprehensive meta-analysis. J Natl Cancer Inst 2005;97:489-98. 
28. Gabrilove JL, Cleeland CS, Livingston RB, et al. Clinical evaluation of once-weekly dosing of epoetin alfa in chemotherapy patients: improvements in hemoglobin and quality of life are similar to three-times-weekly dosing. J Clin Oncol 2001;19:2.8752.882

29. Jones M, Schenkel B, Just J, et al. Epoetin alfa improves quality of life in patients with cancer. Results of a metaanalysis. Cancer 2004;101:1.720-32.

30. Engert A. Oral presentation at Optimizing Hemoglobin Levels and Beyond: Strength for Living and Meaningful Survival? The 3rd Educational Symposium on Advances in Cancer Anemia Management, Vienna, Austria. March 20-23, 2003.

31. Mittelman M, Neumann D, Peled A, et al. Erythropoietin induces tumor regression and antitumor immuneresponses in murine myeloma models. PNAS 2001;98:5.181-6.

32. Rizzo JD, Lichtin AE, Woolf SH, et al. Use of epoetin in patients with cancer: Evidence-based clinical practice guidelines of the American Society of Clinical Oncology and the American Society of Hematology. Blood 2002;100:2.303-2.320.

33. Bokemeyer C, Aapro MS, Courdi A, et al. EORTC guidelines for the use of erythropoietin proteins in anaemic patients with cancer. Eur J Cancer 2004;40:2.201-16.

34. NCCN Clinical Practice Guidelines in Oncology - version.2.2006: Cancer and treatment-related anemia. Available at: www.nccn.org/ professionals/physician_gls/PDF/anemia.pdf

35. Witzig TE, Silverstein PT, Loprinzi CL, et al. Phase III, randomized, double-blind study of epoetin alfa compared with placebo in anemic patients receiving chemotherapy. J Clin Oncol 2005;23:2.6062.617.

36. Shasha D, George MJ, Harrison LB. Once-weekly dosing of epoetin alfa increases hemoglobin and improves quality of life in anaemic cancer patients receiving therapy either concomitantly or sequentially with chemotherapy. Cancer 2003;98:1.072-1.079.

37. Steensma DP, Molina R, Sloan JA, et al. Phase III randomized trial of two different dosing schedules of erythropoietin in anemic patients with cancer. J Clin Oncol 2006;24:1.079-1.089.

38. Waltzman R, Croot C, Justice GR, et al. Randomized comparison of epoetin alfa (40,000 U weekly) and darbepoetin alfa (200 microg every 2 weeks) in anemic patients with cancer receiving chemotherapy. Oncologist 2005;10:642-650.

39. Hedenus M, Hansen S, Taylor $\mathrm{H}$, et al. The Darbepoetin alfa 990114 Study Group. Randomized, dose-finding study of Darbepoetin Alfa in anaemic patients with lymphoproliferative malignancies. Br J Haematol 2002;119:79-86.

40. Glaspy J, Vadhan-Raj S, Patel R, et al. Randomized comparison of every-2-weeks darbepoetin alfa and weekly epoetin alfa for the treatment of chemotherapy-induced anemia: The 20030125 Study Group trial. J Clin Oncol 2006;24:2.290-2.297.

O tema apresentado e o convite ao(s) autor(es) consta da pauta elaborada pelo co-editor.

Avaliação: Co-editor e um revisor externo.

Publicado após revisão e concordância do editor.

Conflito de interesse: não declarado.

Recebido: 25/11/2006

Aceito: 15/01/2007 